\title{
Research on Channel Estimation Algorithm in MIMO-OFDM Technology
}

\author{
Fang Weiwei ${ }^{1}$, Siping $\mathrm{Hu}^{*}, 2$ \\ ${ }^{1}$ Nanyang Institute of Technology, Nanyang, 473000, China \\ ${ }^{2}$ Hubei University of Science and Technology, Xianning, 430070, China
}

Keywords: Orthogonal Frequency Division Multiplexing, power distribution factor, channel estimation, channel estimation

\begin{abstract}
In this paper, by improving the traditional methods in the generation of training sequences and local matrices, a channel estimation method based on superposition training sequences is proposed. The method firstly generates a unipolar non-negative periodic real sequence by performing conjugate symmetric transformation, parallel string transformation, limiting and split combination on the complex sequence; then, according to the time domain statistical characteristics of the received signal and the value of the power distribution factor, the local matrix is reasonably designed; the channel estimation is completed by performing first-order statistical averaging processing on the received signal. Computer simulations show that if the power allocation factor remains the same, as the number of subcarriers in the system increases, the MSE decreases gradually, and the estimated performance is better. As the power allocation factor increases, the energy of the training sequence increases, the better the channel estimation performance and the higher the accuracy, and the effectiveness of the algorithm is verified.
\end{abstract}

\section{Introduction}

Since entering the information age, as the pace of informationization has been accelerating, various information technologies are changing with each passing day, and people are increasingly demanding transmission rates. In the field of optical communication, Wireless Optical (WO) technology can be used for high-rate data transmission. The communication device has a simple structure, low price, no bandwidth limitation, high transmission rate, good security, and is suitable for short-distance high-speed. Wireless access has broad prospects and is developing very rapidly.

It is necessary to estimate the channel in the MIMO-OFDM system, and the accuracy of the channel estimation will directly affect the performance of the whole system. Channel estimation can be divided into: blind channel estimation, pilot-assisted channel estimation, and channel estimation based on superposition sequences, depending on whether additional data needs to be utilized. However, in general, the pilot-based method has higher estimation accuracy and can track time-varying channels, but the pilot occupies spectrum resources and reduces system capacity. The blind channel estimation method does not require pilot information, which is very large. To the extent, the transmission efficiency of the system is improved. However, due to the slow convergence rate, the practical application is limited. The channel estimation method based on the superposition sequence does not require additional frequency bands and time slots to transmit known pilots, which ensures the transmission efficiency of the system and the algorithm. Low complexity and significant advantages. Therefore, the research of channel estimation technology based on superposition sequence in MIMO-OFDM system has more important significance.

\section{Related research}

In 1999, Peter Hoeher and Fredrik Tufvesson used Pseudo Noise (PN) sequences as training sequences, superimposed on the data in the time domain, and used the Viterbi algorithm to implement channel estimation based on superposition sequences for the first time.

Among the channel estimation techniques of the superposition sequence, the most typical is the channel estimation technique of superimposing the periodic sequence. In 2003, G. T. Zhou first 
proposed the use of periodic impulse sequences as superposition sequences, using first-order statistical average method for estimation. Although this method allows a small transmission power, it will generate a large peak-to-average power ratio in the frequency domain, which may overwhelm the data signal on the relevant channel, and is not suitable for the multi-carrier modulation system.

So in 2004, Aldo G. O. used a periodic sequence with a constant modulus as the training sequence, and still used the first-order statistical average method for estimation. This method can optimize the peak-to-average power ratio while achieving accurate channel estimation.

In 2005, M. Ghogho et al. proposed a channel estimation method based on data dependency-based superposition training sequence. In this method, the data in the data symbols with the same frequency as the training sequence is zeroed, thereby completely eliminating the influence of the unknown data on the training sequence, and the receiving end restores the corresponding data by an iterative decision method. This method is similar to the traditional pilot-based channel estimation method, which reduces the system transmission efficiency and has high algorithm complexity.

In 2008, Hashemi S.K. et al. proposed a channel estimation method for indoor wireless optical OFDM systems. The method adjusts the number of frequency domain insertion pilots according to the transformation between the main reflection surface of the scatter channel and the receiver photodetector, so that the channel tracking supports mobility.

\section{Channel estimation algorithm in IMO-OFDM technology}

\subsection{Channel Model Analysis of MIMO-OFDM System}

Wireless optical communication technology can realize wide-angle access of line-of-sight (LOS) and scatter, ie non-line-of-sight (NLOS) links by modulating the intensity of visible or infrared lasers. It is widely used in our real life. In public places such as airplane cabins, train cars and bus cars, as well as public places such as shops, airports and museums, broadband access is provided to users through infrared or LED visible light access points. According to the link topology, the wireless optical channel can be divided into two types of LOS channels and scatter channels.

In the LOS channel, the optical transmitter and receiver are directly aligned, and the light intensity signal is not transmitted through multipath. In the LOS channel, the light intensity signal is mainly affected by the background light, which is subject to a Gaussian distribution, so the LOS channel is usually approximated as a Gaussian channel.

Based on the indoor scatter channel model, the optical transmitter and the receiver are not directly aligned, and the optical signal is reflected multiple times. The optical signal arriving at the receiver comes from different directions, and the signal passes through multipath transmission. The optical signal received by the receiving end is the sum of different optical paths that arrive at the receiver after being reflected by different paths, namely:

$$
r(n)=\sum_{k=0}^{L-1} h(k) x_{c}\left(n-D_{k}\right)+w(n)
$$

The coefficient of the channel impulse response of the first path is the delay time of the signal of the first path relative to the first path signal, indicating AWGN, which is the length of the channel impulse response, that is, the multipath number. The received power of the light intensity signal satisfies the normalized constraint, namely:

$$
\sum_{k=0}^{L-1} h(k)=1
$$

The channel impulse response coefficient of a light scatter channel can usually be accurately described by an exponential decay, ie 


$$
h(k) \frac{e^{-k t_{s} / \tau}}{\sum_{i=0}^{L-1} e^{-i t_{s} / \tau}}, 0 \leq k \leq L-1
$$

\subsection{Channel estimation algorithm}

In order to illustrate the estimation algorithm, the power allocation problem is not considered at present. Processing the received signal sequence, then

$$
y(n)=E[r(k \cdot Q+n)]
$$

From equation (1), equation (4) can be transformed into:

$$
\begin{aligned}
y(n)= & E\left[\sum_{l=0}^{L-1} h(l)\left[s_{c}(k \cdot Q+n-l)+t(k \cdot Q+n-l)\right]+w(k \cdot Q+n)+d\right] \\
= & E\left[\sum_{l=0}^{L-1} h(l) s_{c}(k \cdot Q+n-l)\right]+E\left[\sum_{l=0}^{L-1} h(l) t(k \cdot Q+n-l)\right] \\
& +E[w(k \cdot Q+n)]+E[d], \quad n=0,1, \ldots, Q-1, \quad k=0,1, \ldots, N_{Q}-1
\end{aligned}
$$

Where, it is usually an integer multiple of the training sequence period. Since the mean of the noise sequence is zero, the third term in equation (5) is approximately zero. Since the unknown DC offset is constant, then Equation (5) can be transformed into:

$$
\begin{aligned}
& y(n)=\sum_{l=0}^{L-1} h(l) B+\sum_{l=0}^{L-1} h(l) t(k Q+n-l)+d \\
&=\sum_{l=0}^{L-1} h(l)[B+t(k Q+n-l)]+d \\
&=\sum_{l=0}^{L-1} h(l)[B+t(n-l)]_{Q}+d, \quad n=0,1, \ldots, Q-1, \quad k=0,1, \ldots, N_{Q}-1
\end{aligned}
$$

The condition that the formula (6) has a unique solution is that the training sequence period must be equal to the channel impulse response order, and its coefficient matrix is a full rank matrix. Therefore, the channel order must be accurately obtained, but this cannot be accurately obtained in the actual situation, and only the upper limit can be estimated. Thus, the training sequence period is equal to the maximum value that the channel order can achieve, and the channel impulse response coefficient can be solved. Assume that the system does not have an unknown DC offset. Express equation (6) as a matrix:

$$
\mathbf{T h}=\mathbf{y}
$$

Since the sample sequence length is a finite value within an MIMO-OFDM symbol period and the sequence distribution has a stationary ergodicity, the unbiased estimate of $\mathbf{y}$ is:

$$
\hat{y}(n)=\frac{1}{N_{Q}} \sum_{k=0}^{N_{Q}-1} r(k \cdot Q+n), \quad n=0,1, \ldots, Q-1
$$

Thus, the channel estimate can be done by:

$$
\hat{\mathbf{h}}=\mathbf{T}^{-1} \hat{\mathbf{y}}
$$

\section{Simulation analysis}

In this paper, the performance of the channel estimation method based on superimposed periodic 
sequence of ACO-OFDM wireless optical communication system proposed in this paper is simulated in wireless light scattering channel using MATLAB 7.6.0 simulation software. The feasibility of this method and the previous section are verified. The correctness of the theoretical analysis. According to the principle model of the ACO-OFDM system, the source program is written by using the least squares estimation algorithm.

Here we use 4QAM digital modulation method; the PN sequence consists of a constant-mode sequence of length 4, after splitting and combining, cyclically repeating a unipolar non-negative periodic real sequence with a period of 8 ; MIMO-OFDM system subcarrier number; CP Length, $N_{C P}=N / 8$. This study is based on the indoor wireless light scatter channel transmission model. The adjacent path delay time is equal to the system sampling interval, the channel impulse response length is $\mathrm{L}=6$, and the channel impulse response coefficient. Starting from the time saving, the simulation results of this paper are based on 1000 Monte Carlo simulations.

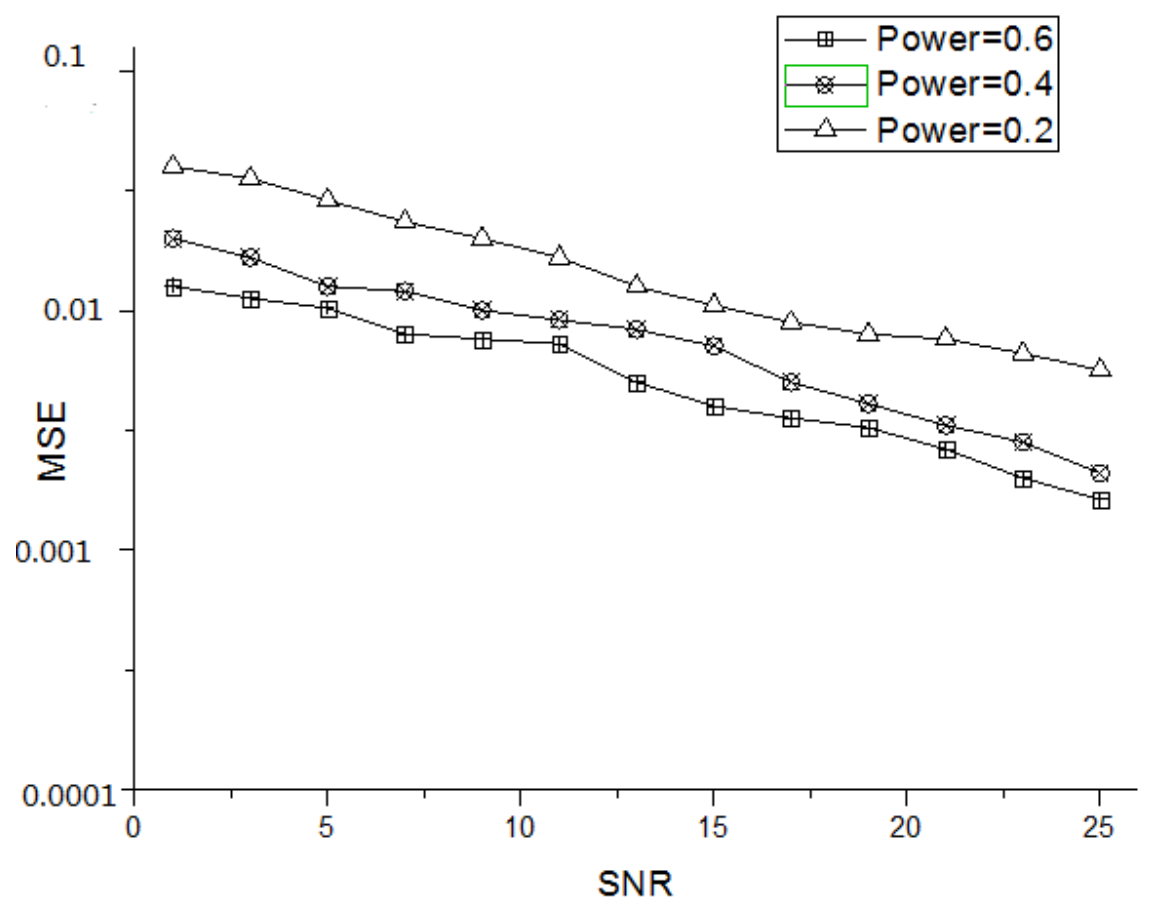

Fig. 1 Influence of power allocation factor on channel estimation performance

In practical systems, there are many factors that influence the choice of power distribution factors. The contradiction between the training sequence and the system image response and channel estimation performance must be weighed in the selection process. Under the premise of satisfying certain system BER requirements and average transmit power, the transmission efficiency of the data symbols superimposed by the training sequence can be changed by adjusting the size of the training sequence power allocation factor. The larger the power allocation factor, the lower the system transmission efficiency; the smaller the power distribution factor, the higher the system transmission efficiency. Figure 1 shows the simulation of the impact of the power distribution factor on system performance. It can be seen that the system performs best when it is between 0.3 and 0.5 .

\section{Conclusion}

In this paper, the development background and research status of superposition sequence channel estimation technology are briefly described. Then, according to the technical characteristics of ACO-OFDM wireless optical communication system, a channel estimation method based on superposition periodic sequence is applied. At the same time, the channel model of ACO-OFDM system is analyzed. For the system channel estimation method, we mainly do the following three aspects: training sequence generation, local matrix generation and channel estimation algorithm 
research. Then through the theoretical derivation, the performance of the proposed method is analyzed. Finally, the effectiveness of the proposed scheme and the correctness of the theoretical analysis are verified by the comprehensive simulation of the system, and the practicability of the channel estimation algorithm is demonstrated.

\section{Acknowledgements}

This research was supported by key scientific research projects of universities in Henan (16A510007), Henan science and technology project(182102210459), Young backbone teacher Project of Nanyang Institute of Technology(50104026), Doctor Initial Funding of Hubei University of Science and Technology (No. 2016-19XB003), the Scientific Research Project of Education Department of Hubei Province under Grant B2018179 and B2017181 and B2018175, the National Natural Science Foundation of China (No. 51479155).

\section{References}

[1] S. C. J. Lee, F. Breyer, S. Randel, M. Schuster, J. Zeng, F. Huiskens, H. P. A. vanden Boom, A. M. J. Koonen, N. Hanik. 24-Gb/stransmission over $730 \mathrm{~m}$ of multimode fiber by direct modulation of 850-nm VCSEL using discrete multi-tone modulation. the Proc. OFC/NFOEC 2007 [C]. Anaheim, CA. 2007. PDP6.

[2] N. Cvijetic, D. Qian, T. Wang. $10 \mathrm{~Gb} / \mathrm{s}$ free-space optical transmission using OFDM [A]. the Proc. OFC/NFOEC 2008. San Diego, CA. 2008. OTHD2.

[3] ARMSTRONG J, SCHMIDT B J C. Comparison of Asymmetrically Clipped Optical OFDM and DC-Biased Optical OFDM in AWGN. Communications Letters, IEEE, 2008, 12(5): 343-5.

[4] WILSON S K, ARMSTRONG J. Transmitter and receiver methods for improving asymmetrically-clipped optical OFDM. Wireless Communications, IEEE Transactions on, 2009, 8(9): 4561-7.

[5] MOREOLO M S, MUOZ R, JUNYENT G. Novel Power Efficient Optical OFDM Based on Hartley Transform for Intensity-Modulated Direct-Detection Systems. Lightwave Technology, Journal of, 2010, 28(5): 798-805.

[6] Aldo G. O., Mauricio M. L., McLernon D. C.. Channel estimation using implicit training. IEEE Transactions on Signal Processing. 2004, 52(1):240-254. 